\title{
Effect of the administration of Psidium guava leaves on blood glucose, lipid profiles and sensitivity of the vascular mesenteric bed to Phenylephrine in streptozotocin-induced diabetic rats
}

\author{
Abdol Hassan Mansoori Bahrani ${ }^{1}$, Habib Zaheri ${ }^{2}$, Nepton Soltani ${ }^{i^{*}}$, Fatemeh Kharazmi ${ }^{3}$, \\ Mansoor Keshavarz ${ }^{4}$, Mohammad Kamalinajad ${ }^{5}$ \\ ${ }^{1}$ Department of Science, Minab, Islamic Azad University, Minab, Iran \\ ${ }^{2}$ Department of Biology, Faculty of Science, Shahid Bahonar University, Kerman, Iran \\ ${ }^{3}$ Department of Physiology, School of Medicine and Cardiovascular Research Center, Hormozgan University of Medical Sciences, \\ Bandar Abbas, Iran; ${ }^{*}$ Corresponding Author: neptun.soltani@gmail.com, nsoltani@hums.ac.ir \\ ${ }^{4}$ Department of Physiology, Faculty of Medicine, Tehran University of Medical Sciences, Tehran, Iran \\ ${ }^{5}$ Department of Pharmacognosy, Faculty of Pharmacy, Shahid Bahashti University of Medical Sciences, Tehran, Iran
}

Received 25 November 2011; revised 27 December 2011; accepted 10 January 2012

\section{ABSTRACT}

The leaf of Psidium guava is traditionally used in Asia to manage, control and treat diabetes. We designed this study to elucidate the effect of the administration of oral doses of aqueous and ethanol extract from Psidium guava leaves on plasma glucose, lipid profiles and the sensitivity of the vascular mesenteric bed to Phenylephrine in diabetic and non diabetic rats. Animals were divided into 5 groups $(n=10)$ : two groups served as non-diabetic controls (NDC), while the other groups had diabetes induced with a single injection of streptozotocin (STZ). Psidium guavatreated chronic diabetic (PSG-CD) and Psidium guava-treated controls (PSG-C) received $1 \mathrm{~g} / \mathrm{l}$ of Psidium guava added to the drinking water for 8 weeks. The mesenteric vascular beds were prepared using the McGregor method. Administration of Psidium guava caused $\mathrm{Ca} / \mathrm{Mg}$ ratio, plasma glucose, high density lipoprotein (HDL), low density lipoprotein (LDL), very low density lipoprotein (VLDL), total cholesterol and triglyceride concentrations to return to normal levels, and was shown to decrease alteration in vascular reactivity to vasoconstrictor agents. Our results support the hypothesis that Psidium guava could play a role in the management of diabetes and the prevention of vascular complications in STZ-induced diabetic rats.

Keywords: Diabetes; Lipid Profile; Psidium guava; Blood Glucose; Mesenteric Bed; Ca/Mg Ratio

\section{INTRODUCTION}

Diabetes mellitus is a type of metabolic disorder that is characterized by hyperglycemia and alterations in carbohydrate, fat and protein metabolisms associated with absolute or relative deficiencies in insulin secretion and/ or insulin action [1]. Approximately one-third of patients with type 1 diabetes will develop related complications [2]. The causes are not completely understood, but a more sedentary lifestyle, the consumption of an energy rich diet, obesity, a longer life span [3], long-term diabetes, poor glycemic control, hypertension and dyslipidemia are linked to diabetes-related vascular complications [4]. Diabetes is characterized by a high incidence of cardiovascular disease [5]. There has been increasing evidence recently that postprandial diabetes and hyperglycemia are important contributory factors in atherosclerosis [5]. In diabetes, the postprandial phase is characterized by a massive rapid increase in blood glucose levels where alteration in the sensitivity or reactivity of vascular smooth muscle to neurotransmitters and circulating hormones may cause or contribute to diabetic vessel complications [5,6]. Clinical atherosclerotic disease is positively associated with total cholesterol and LDL cholesterol levels, while it is inversely associated with HDL cholesterol [7].

The search for appropriate hypoglycemic agents has recently been focused on plants and many herbal medicines have been recommended for the treatment of diabetes [8]. Herbal drugs are frequently considered to be less toxic than their synthetic counterparts and they have fewer side effects [9]. The leaf of Psidium guava is traditionally used in Asia and other countries to manage con- 
trol and treat diabetes. In other parts of the world, the leaves of Psidium guava are used to treat many other human ailments including caries, pyrexia, cough, wounds, bleeding gums, inflammation, epilepsy, kidney problems and ulcers [10]. In Japan, Guava leaf tea containing the aqueous leaf extract has been approved for specific health uses and is now commercially available $[11,12]$.

However, little is known about the therapeutic action, mechanism or safety of these treatments in healthy human beings. Some studies have shown administration of Psidium guava leaves decreases blood glucose via enhanced insulin secretion [13]. Cheng et al. suggest that quercetin in the aqueous extract of guava leaves promotes glucose uptake in liver cells and consequently contributes to the alleviation of hypoglycemia in diabetes [14]. Shen et al. [1] and Ojewole [10] have provided evidence to support the antihyperglycemic effect of guava leaf extract and the positive action of guava leaves against type 2 diabetes, whereas Sungawa et al. [15] showed Psidium guava leaves to have no effect on blood glucose.

Other researchers have found Psidium guava leaves to have an effect on blood glucose but not on lipid profiles and diabetic vessels. Olatunji-Bello et al. [16] demonstrated the contractile effect of Psidium guava on normal isolated rat aorta rings while Witness et al. [11] observed a vasorelaxant effect of Psidium guava on aortic rings in normal rats.

In view of the conflicting outcomes of these reports, we designed this study to elucidate the effect of oral administration of Psidium guava leaves on plasma glucose, triglycerides, LDL, HDL, VLDL, total cholesterol levels and the sensitivity of the vascular mesenteric bed to Phenylephrine in normal and diabetic rats.

\section{MATERIALS AND METHODS}

\subsection{Animals}

The animals were handled in accordance with the criteria outlined in the "Guide for the Care and Use of Laboratory Animals" (http://www.nap.edu/readingroom/ books/labrats/). Male Wistar rats, weighing 180-250 g were selected and maintained at a constant temperature of $22^{\circ} \mathrm{C} \pm 2{ }^{\circ} \mathrm{C}$ with a fixed $12: 12$-h light-dark cycle. Nutritionally balanced pellets and water were freely available. The animals were divided into five groups (10 rats in each group): non-diabetic control (NDC); acute diabetic (AD); chronic diabetic (CD); Psidium guavatreated chronic diabetic (PSG-CD), and; Psidium guavatreated control (PSG-C).

Diabetes was induced with and IP injection of 60 $\mathrm{mg} / \mathrm{kg} \mathrm{STZ}$. At 10 days after diabetes induction, fasting blood glucose level was determined and the presence of diabetes was confirmed by blood glucose levels of above $250 \mathrm{mg} / \mathrm{dl}$. Animals in which the diabetes lasted for 10 days were classed as "acute diabetic" and those in which the diabetes lasted for 8 weeks were defined as "chronic diabetic". The PSG-CD and PSG-C groups received 1g/1 of aqueous and ethanol extract of Psidium guava leaf added to the drinking water, 10 days after diabetes induction, for 8 weeks. Animals were monitored for blood glucose concentrations and body weight every week over the course of the experiment. Blood glucose was measured with an Ascensia ELITE XL glucometer and Ascensia Elite blood glucose test strips.

\subsection{Preparation of Guava Leaf Extract}

Fresh leaves of Psidium guava were collected from open grassland in Menab (Southern Iran). The plant was identified by the Taxonomist at the ShahidBeheshti University of Medical Sciences. A kilogramme of fresh Psidium guava leaves was air dried under shade at room temperature $\left(26^{\circ} \mathrm{C} \pm 1{ }^{\circ} \mathrm{C}\right)$ for 2 weeks. The dried leaves were then milled into a fine powder in a Waring commercial blender. The powdered leaves were extracted with 3 volumes of $95 \%$ ethanol for 3 days. The extract was filtered through filter paper (Whatman No. 1) and concentrated with a rotary vacuum evaporator (Eyela N-3000 Nseries, Tokyo Rikakikai, Tokyo, Japan). The concentrate was then freeze-dried (Model 77500-00 M, Labconco Co., MO) and stored at $-80^{\circ} \mathrm{C}$ before use as per Shen et al. [1]. One gram of Psidium guava leaf extract was contain $75.7 \mathrm{mg}$ dried substance.

\subsection{Intraperitoneal Glucose Tolerance Test (IPGTT)}

For IPGTT, animals in the NDC, CD and PSG-CD groups were fasted overnight for $15 \mathrm{~h}$, and were given $1.5 \mathrm{~g} / \mathrm{kg}$ body weight glucose via IP injection. Blood was drawn from the tail vein at $0,10,20,30,60,90$ and 120 min after glucose administration.

\subsection{Biochemical Assay}

Animals were decapitated after anesthesia induction with ketamine $\mathrm{HCl}$. Blood samples were taken from the neck vascular trunk in order to determine the triglycerides, total cholesterol, HDL-cholesterol, magnesium and calcium levels by spectrophotometer (UV 3100, Shimadzu) and appropriate kits (Zistshimi, Tehran, Iran). LDL and VLDL cholesterol were calculated by the following formulae as per Saunders et al. (1999) [17]:

VLDL $=$ Triglycerides $/ 5$

LDL $=$ Total cholesterol - HDL cholesterol - VLDL 


\subsection{Preparation of the Mesenteric Vascular Bed}

The animals in the NDC, CD and PSG-CD groups were anesthetized by IP injection of ketamine $\mathrm{HCl} 50$ $\mathrm{mg} / \mathrm{kg}$ and the mesenteric vascular beds were prepared as originally described by McGregor (1965) [18]: the abdominal wall was opened, the superior mesenteric artery was exposed and cannulated, then gently flushed with modified Krebs-Henseleit solution (containing [in $\mathrm{mM}$ ]: $\mathrm{NaCl}: 118, \mathrm{KCl}: 4.7, \mathrm{CaCl}_{2} ; 2.5, \mathrm{MgSO}_{4} ; 1.2$, glucose; 2 , $\left.\mathrm{NaHCO}_{3} ; 2.5, \mathrm{NaHPO}_{4} ; 1.2\right)$ concomitantly bubbled with a mixture of $95 \% \mathrm{O}_{2}$ and $5 \% \mathrm{CO}_{2}$ (final $\mathrm{pH} 7.4$ ), and warmed to $37^{\circ} \mathrm{C}$. The mesentery was isolated from the intestine and placed in a water-jacketed perfusion chamber maintained at $37^{\circ} \mathrm{C}$. The preparation was perfused at $1 \mathrm{ml} / \mathrm{min}$ with modified Krebs-Henseleit solution by a peristaltic pump (Meredos $\mathrm{GmbH}$ ). The tissue was prevented from drying by superfusion with $0.1 \mathrm{ml} / \mathrm{min}$ modified Krebs-Henseleit solution. Perfusion pressure was monitored via a T-tube inserted between the pump and the inflow cannula. This was connected to a pressure transducer (MLT0380 AD Instruments). The recording was performed by Power Lab System (16SP, AD Instruments). Tissues were used for experiment after equilibration of 30-minutes. After the 30-minute equilibration, the vascular bed was constricted by Krebs-Henseleit solution containing phenylephrine, an $\alpha_{1}$-adrenoceptor agonist, from $0.0001 \mathrm{M}$ to $0.1 \mathrm{M}$ and perfusion pressure was recorded. Drug concentrations were increased every 15 minutes.

\subsection{Drugs}

The following drugs were used: STZ from Sigma
(USA) dissolved in $1 \mathrm{ml}$ normal saline immediately before use. Phenylephrine from Sigma (St. Louis, MO, USA) and ketamine $\mathrm{HCl}$ from Rotexmedica (Trittau, Germany).

\subsection{Statistical Analysis}

Data were expressed as mean \pm S.E.M. Differences among groups were evaluated by one-way and two-way ANOVA with the Tukey post-hoc test. $\mathrm{P}<0.05$ was selected for acceptance of statistical significance.

\section{RESULTS}

Before the induction of diabetes, there were no differences among the animals in each group.

\subsection{Changes in Plasma Glucose}

Changes in feeding plasma glucose were measured in all groups (Figure 1). Diabetes induction caused plasma glucose concentration to increase $(440 \pm 71.99)$ and blood glucose continued to be elevated $(442.5 \pm 58.93) 8$ weeks after diabetes induction. Administration of Psidium guava for 8 weeks (from day 10) caused the plasma glucose concentrations in the PSG-CD group to return to normal levels $(118.88 \pm 9.44)$ but there were no changes in blood glucose in the PSG-C group.

\subsection{Effect of Psidium guava Leaf Extracts on Intraperitoneal Glucose Tolerance Test (IPGTT)}

Before initiation of Psidium guava and STZ administration, the IPGTT patterns for 3 groups (NDC, CD and

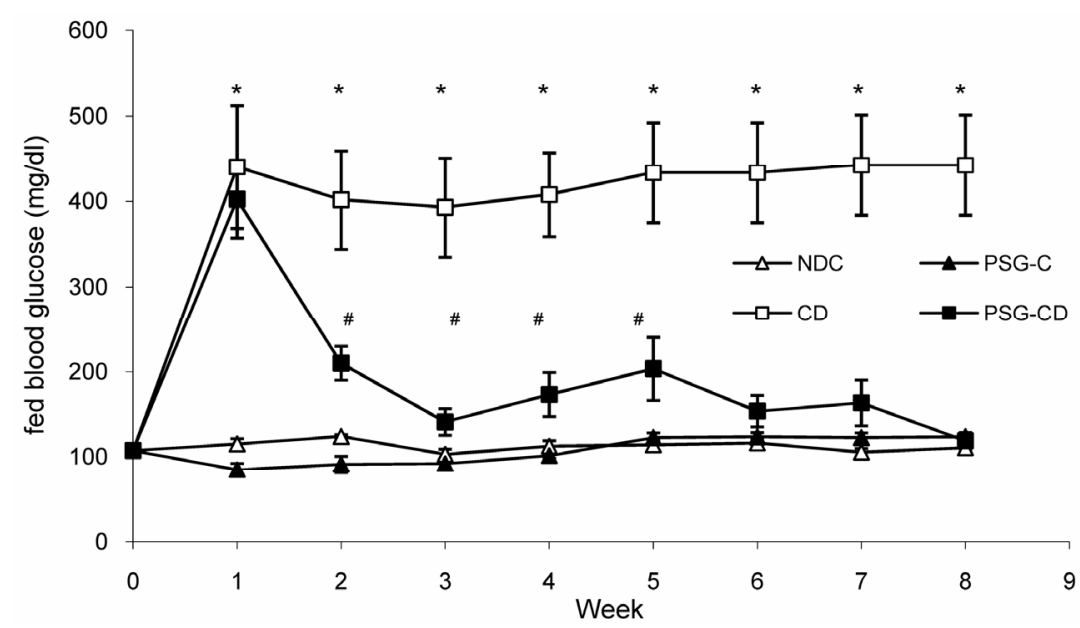

Figure 1. Comparison of fed blood glucose in non-diabetic control (NDC), acute diabetic (AD), chronic diabetic (CD), Psidium guava-treated control (PSG-C) and Psidium guava-treated chronic diabetic (PSG-CD) groups (10 rats in each group. data are expressed as mean \pm SEM). * Significant difference between chronic diabetic and other groups $(\mathrm{P}<0.0001)$; ${ }^{\#}$ Significant difference between Psidium guava-treated chronic diabetic and NDC groups $(\mathrm{P}<0.05)$. 
PSG-CD) were similar and there was no significant difference between the groups when the glycemic response was expressed as the area under the curve (AUC) (data was not shown). However, after 8 weeks, the CD group displayed severe glucose intolerance, which was significantly improved in the PSG-CD group to a degree that was judged effective (Figure 2(b), AUC: NDC vs. CD vs. PSG-CD $=26606 \pm 1430.9$ vs. $55273.5 \pm 6764.68$ vs. $20627 \pm 1894.27 \mathrm{P}<0.0001)$.

\subsection{Changes in Plasma Calcium, Magnesium and $\mathrm{Ca} / \mathrm{Mg}$ Ratio}

Changes in plasma calcium $(\mathrm{Ca})$, magnesium $(\mathrm{Mg})$ and the $\mathrm{Ca} / \mathrm{Mg}$ ratio were measured in all groups (Figures 3(a) to 3(c)) and 8 weeks after the administration of Psidium guava leaves the plasma calcium level in the PSG-CD group was unchanged in comparison with the

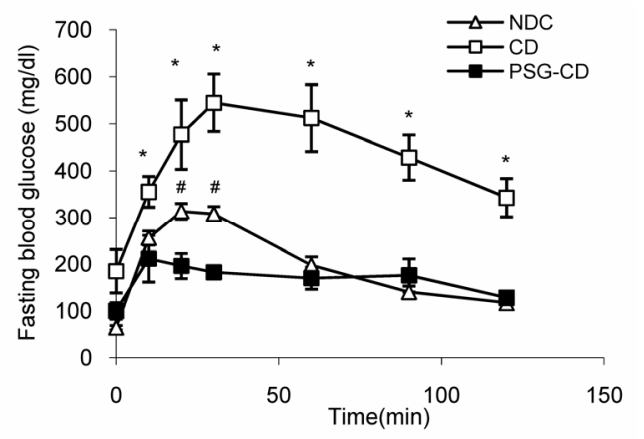

(a)

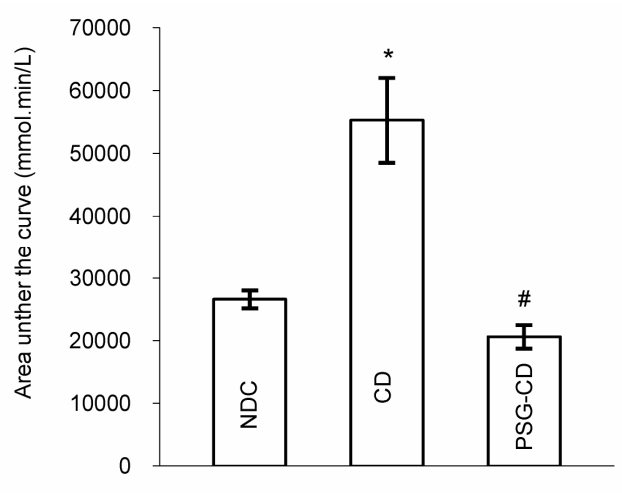

(b)

Figure 2. Comparison of intraperitoneal glucose tolerance test (IPGTT) (a) and the area under the glycaemic curve (b) in non-diabetic control (NDC), acute diabetic (AD), chronic diabetic (CD), Psidium guava-treated control (PSG-C) and Psidium guavatreated chronic diabetic (PSG-CD) groups (10 rats in each group. data are expressed as mean $\pm \mathrm{S}$ EM). *Significant difference between chronic diabetic and other groups $(\mathrm{P}<0.0001)$; "Significant difference between Psidium guava-treated chronic diabetic and NDC groups $(\mathrm{P}<0.05)$.

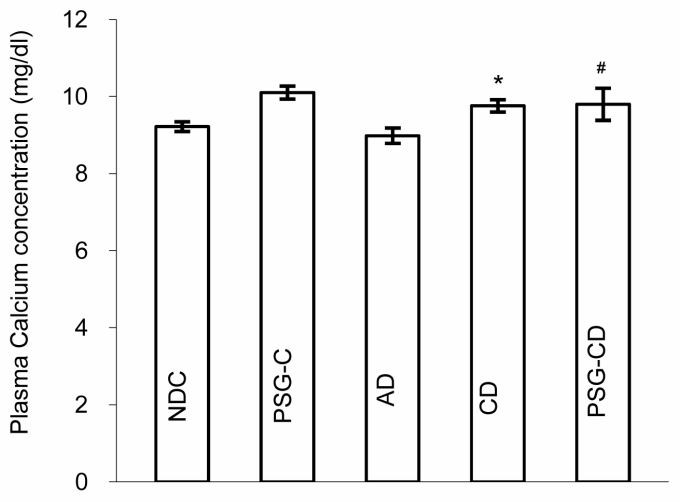

(a)

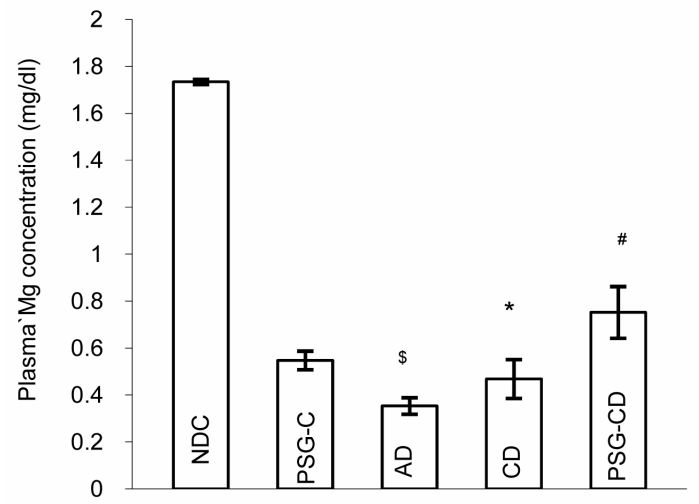

(b)

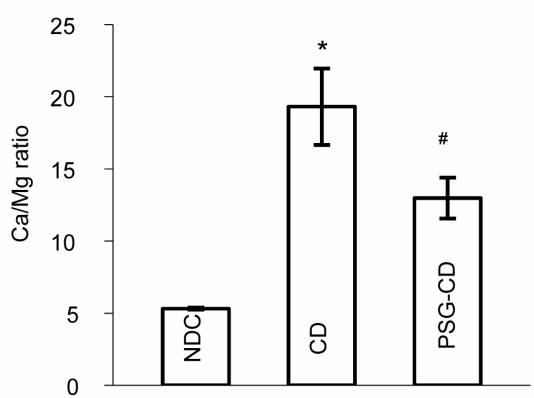

(c)

Figure 3. Plasma Calcium (a) and Magnesium (b) concentrations and $\mathrm{Ca} / \mathrm{Mg}$ ratio (c) in non-diabetic control (NDC), acute diabetic (AD), chronic diabetic (CD), Psidium guava-treated control (PSG-C) and Psidium guava-treated chronic diabetic (PSG-CD) groups (10 rats in each group, data were expressed as mean \pm SEM). *Significant difference between CD and NDC group $(\mathrm{P}<$ 0.001); "Significant difference between PSG-CD and other groups $(\mathrm{P}<0.001)$; ${ }^{\text {S Significant difference between }}$ $\mathrm{AD}$ and $\mathrm{PSG}-\mathrm{C}$ with other groups $(\mathrm{P}<0.001)$.

NDC and CD groups (NDC vs. AC vs. CD vs. PSG-CD $=9.22 \pm 0.12$ vs. $8.98 \pm 0.19$ vs. $9.76 \pm 0.16$ vs. $9.7 \pm$ 0.41 vs.). However a significant increase $(\mathrm{p}<0.001)$ was seen in the PSG-C group $(10.1 \pm 0.16)$ in comparison to 
the NDC group (Figure 3(a)).

The plasma magnesium level $(0.35 \pm 0.035)$ significantly $(\mathrm{P}<0.001)$ decreased compared to the NDC group $(1.73 \pm 0.01) 10$ days after diabetes induction and the plasma magnesium level was still decreased $(0.46 \pm 0.08)$ 8 weeks after diabetes induction. Administration of Psidium guava for 8 weeks (from day 10) caused plasma magnesium concentrations in the PSG-CD group to increase although these did not reach normal levels $(0.75 \pm$ $0.11)$. It is interesting that Psidium guava administration in the PSG-C group $(0.54 \pm 0.03)$ caused the plasma magnesium level to decrease significantly $(\mathrm{P}<0.001)$ in comparison to the NDC group.

The $\mathrm{Ca} / \mathrm{Mg}$ ratio in the $\mathrm{CD}$ and $\mathrm{PSG}-\mathrm{C}$ groups increased significantly $(\mathrm{P}<0.001) 8$ weeks after induction of diabetes compared to the NDC group (NDC vs. AC vs. CD vs. PSG-C vs. $\mathrm{PSG}-\mathrm{CD}=3.31 \pm 0.07$ vs. $23.32 \pm 1.6$ vs. $19.3 \pm 2.65$ vs. $18.5 \pm 1.21$ vs. $12.97 \pm 1.41)$.

\subsection{Changes in Plasma Lipid Profiles}

Changes in plasma triglycerides, total cholesterol, HDL, LDL and VLDL concentrations were measured in all groups (Figures 4(a) to 4(e)). LDL, VLDL, total cholesterol and triglyceride concentrations were elevated significantly $(\mathrm{P}<0.01)$ in the diabetic control group 8 weeks after induction of diabetes, while HDL concentration decreased significantly $(\mathrm{P}<0.01)$ compared to the NDC group. Administration of Psidium guava for 8 weeks (from day 10) caused plasma VLDL and triglyceride concentrations to return to normal levels but LDL and total cholesterol levels were lower than in the NDC group. The plasma HDL level in PSG-CD animals was significantly higher than in the NDCs, but plasma HDL in the PSG-C group was lower than in the NDC group.

\subsection{Mesenteric Bed Response}

Baseline perfusion pressure for the $\mathrm{CD}$ group was significantly $(p<0.001)$ higher than for the NDC and PSG$\mathrm{CD}$ groups, but there was no significant difference between NDC animals and the PSG-CD group (Figure 5).

After equilibration of 20 minutes, a cumulative concentration-response curve was plotted for phenylephrine, an $\alpha 1$ adrenoceptor agonist, (from 0.0001 to $0.1 \mathrm{M}$ ) in intact mesenteric beds. Phenylephrine was added into the medium and perfusion pressure (15 min for each concentration) was recorded (Figure 6). The phenylephrine induced concentration-dependent vasoconstriction in mesenteric beds manifested as an increase in perfusion pressure (Figure 6). Maximum vasoconstriction was achieved at Phenylephrine concentration of $0.01 \mathrm{M}$.

Contractile responses to Phenylephrine at concentrations higher than $0.006 \mathrm{M}$ were found to be significantly increased in the mesenteric beds of $\mathrm{CD}$ rats compared to the NDC and PSG-CD groups. However, there was no significant difference between the NDC and PSG-CD groups at high doses of Phenylephrine.

\section{DISCUSSION}

This study was designed to investigate the effect of the oral administration of Psidium guava leaf extract on plasma glucose, triglycerides, LDL, HDL, VLDL total cholesterol levels and the sensitivity of the vascular mesenteric bed to Phenylephrine in normal and diabetic rats.

The results of the present study have shown that administration of $1 \mathrm{~g} / \mathrm{l}$ of Psidium guava leaf extract to drinking water of diabetic animals for 8 weeks may decrease blood glucose levels. The mechanism is not well understood. The compositions of Psidium guava leaf extract are including alkaloids, anthocyanins, carotenoids,essential oils, fatty acids, lectins, phenols, saponins, tannins, triterpenes, and vitamin C (80 mg per $100 \mathrm{~g}$ of guava), quercetin, flavonoids, morin and myricetin. The main active constituent in the plant is reported to be flavonoids $[11,19]$.

Obatomi et al. [13] showed Psidium guava to decrease blood glucose levels via an increase in insulin secretion, while Sunagawa et al. [15] showed Psidium guava to increase blood glucose levels. IPGTT outcomes from the present study could lead us to believe that Psidium guava may repair pancreas beta cells and enhance insulin secretion that then decrease blood glucose levels. Furthermore, treatment with Psidium guava improves glucose tolerance in diabetic rats, suggesting an enhanced insulin secretion. Blood glucose was unable to reach normal levels in the untreated diabetic rats 2 hours after glucose injection, while the blood glucose in the Psidium guavatreated diabetic rats returned to normal levels.

In the present study, plasma magnesium levels increased after Psidium guava administration but did not attain the same levels as in the NDC group. Intracellular magnesium shift may explain this observation in line with our previous studies [20-22]. It is interesting, however, that Psidium guava administration in PSG-C animals may decrease plasma magnesium levels. Our previous studies [20-22] have shown magnesium deficiency as a potential factor in the pathogenesis of complications in diabetes and it appears that human consumption of Psidium guava in foodstuffs may increase the risk of diabetes induction.

Our finding showed the $\mathrm{Ca} / \mathrm{Mg}$ ratio to be significantly elevated in untreated diabetic rats while it decreased in the Psidium guava treated group. $\mathrm{Ca} / \mathrm{Mg}$ ratio is a marker of vascular tone where an increase represents increased vascular reactivity and atherogenic risk [23]. Atherogenic lesion is poorly correlated with serum cho- 


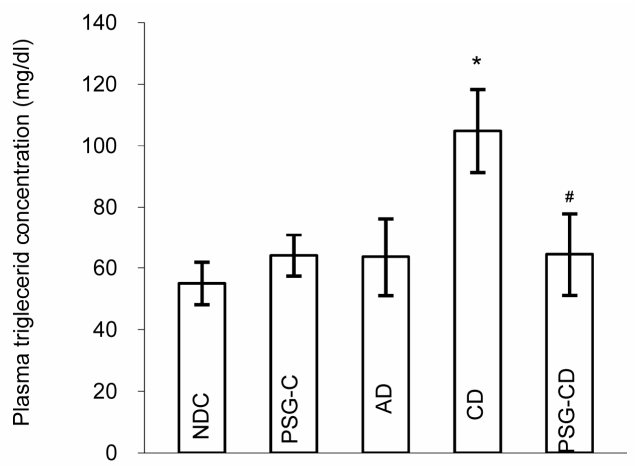

(a)

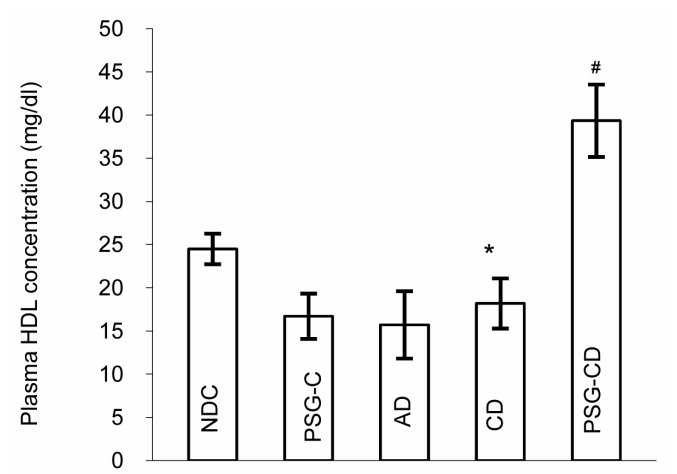

(c)

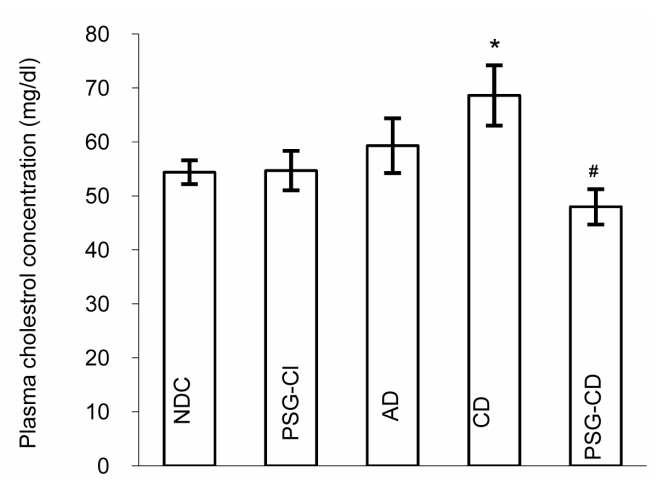

(b)

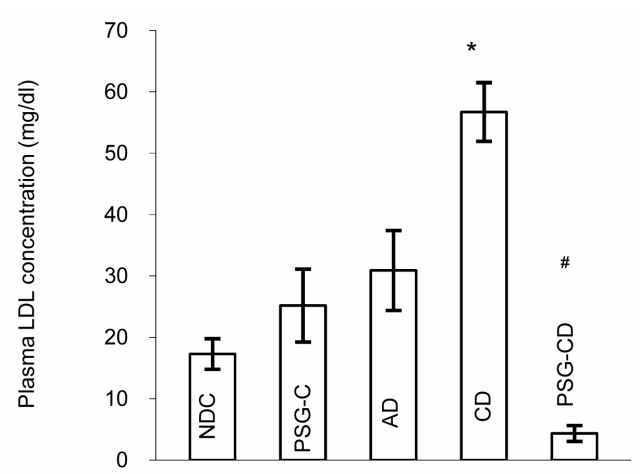

(d)

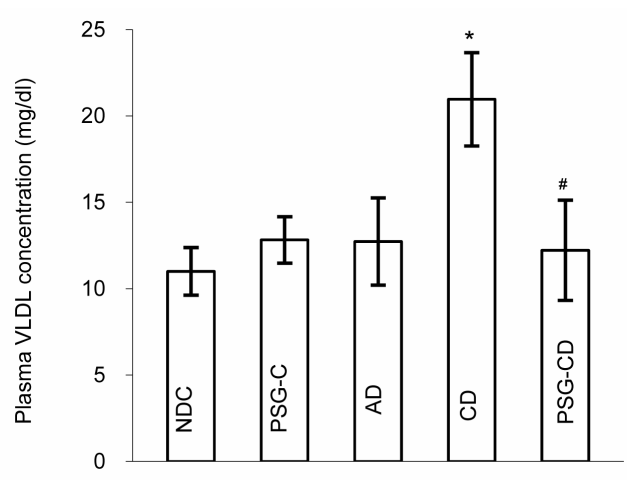

(e)

Figure 4. Plasma triglyceride (a), cholesterol (b), HDL (c), LDL (d), VLDL (e) concentrations in non-diabetic control (NDC), acute diabetic (AD), chronic diabetic (CD), Psidium guava-treated control (PSG-C) and Psidium guava-treated chronic diabetic (PSG-CD) groups (10 rats in each group, data were expressed as mean \pm SEM). " Significant difference between $\mathrm{CD}$ and NDC groups $(\mathrm{P}<0.01)$; ${ }^{\text {\# }}$ Significant difference between PSG-CD and other groups $(\mathrm{P}<0.01)$.

lesterol level and highly dependent on plasma magnesium level and $\mathrm{Ca} / \mathrm{Mg}$ ratio [23]. Therefore Psidium guava administration can decrease the $\mathrm{Ca} / \mathrm{Mg}$ ratio and prevent atherosclerosis.

Our results show that Psidium guava administration could decrease triglycerides, cholesterol, VLDL and LDL cholesterol and also increase HDL cholesterol. The decrease in serum triglycerides is associated with the change in total serum $\mathrm{Mg}$ concentration. There is in- creasing evidence for the role of magnesium in the modulation of serum lipids and lipid uptake in macrophages [24].

Vascular disease is one of the complicating features of diabetes mellitus. Several prospective studies have indicated that hypertension in diabetic patients occurs at twice the rate for the non-diabetic population [25]. Hypertension is also considered an independent risk factor for cardiovascular mortality in patients with diabetes [25]. 


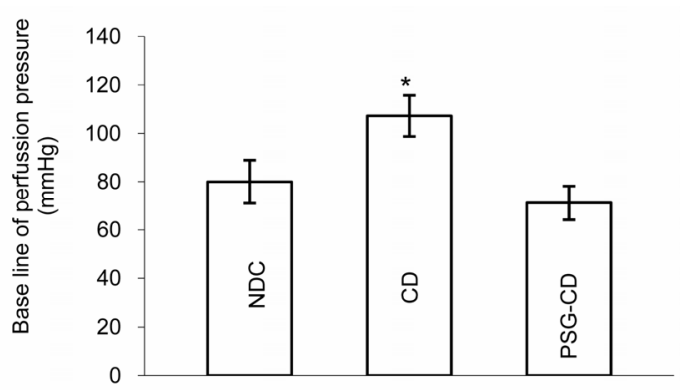

Figure 5. Baseline perfusion pressure $(\mathrm{mmHg})$ of mesenteric vascular bed in non-diabetic control (NDC), chronic diabetic (CD) and Psidium guava-treated chronic diabetic (PSG-CD) rats with intact endothelium (6 rats in each group, data expressed as Mean $\pm \mathrm{SEM}) ;{ }^{*}$ Significant difference between $\mathrm{CD}$ and other groups $(\mathrm{P}<0.001)$.

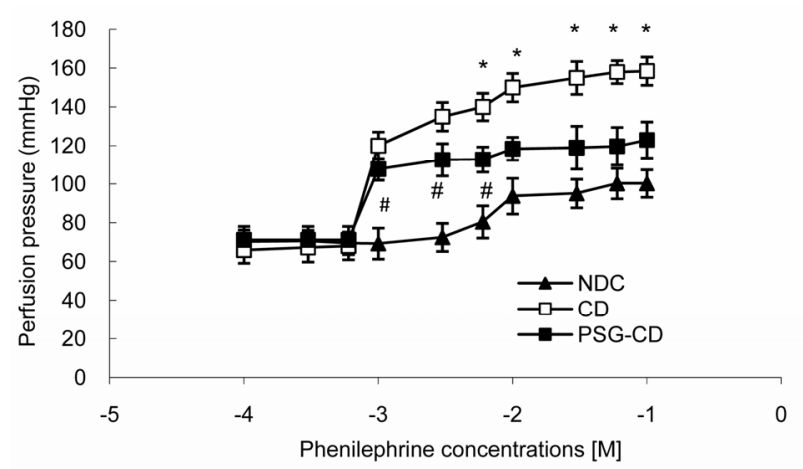

Figure 6. Dose response curve of Phenylephrine in mesenteric vascular bed with intact endothelium in non-diabetic control (NDC), chronic diabetic (CD) and Psidium guava-treated chronic diabetic (PSG-CD) animals. (6 rats in each group, data expressed as Mean \pm SEM); ${ }^{*}$ Significant difference between CD and other groups $(\mathrm{P}<0.001)$; ${ }^{\#}$ Significant difference between PSG-CD and NDC groups $(\mathrm{P}<0.001)$.

It has been suggested that alterations in the reactivity of blood vessels to neurotransmitters and circulating hormones are responsible for the cardiovascular complications of diabetes [6]. In our study, response to phenylephrine was considered an alteration in vascular reactivity to vasoconstrictor agents, and alteration in the $\mathrm{Ca} / \mathrm{Mg}$ ratio was considered a marker for atherosclerosis. Our results show that the administration of Psidium guava for 8 weeks could decrease the alteration in vascular reactivity to vasoconstrictor agents and also decrease vessel atherosclerosis.

In conclusion, we have observed that Psidium guava administration can improve lipid profile and decrease blood glucose levels, $\mathrm{Ca} / \mathrm{Mg}$ ratio and vascular reactivity to vasoconstrictor agents. It can also decrease vessel atherosclerosis and prevent diabetic vessel complications. In our view, it appears that Psidium guava administration may decrease cardiovascular risk factors.

Our results support the hypothesis that Psidium guava has a potential role in the management of diabetes and in the prevention of some complications in STZ-induced diabetic rats and that it maybe useful in the treatment of hyperlipidemia in diabetes. Further studies will be needed to confirm the role of Psidium guava in lipid metabolism control.

\section{ACKNOWLEDGEMENTS}

The authors would like to thank the Deputy of Research of Hormozgan University of Medical Sciences and we are also deeply appreciative of the help provided by Mr. MustafaKazemi in the physiology department of animal room. We thank S. Griffin-Mason (AuthorAID in the Eastern Mediterranean)for improving the use of English in the manuscript.

\section{REFERENCES}

[1] Shen, S.C., Cheng, F.C. and Wu, N.J. (2008) Effect of Guava (Psidiumguajava Linn.) leaf soluble solids on glucose metabolism in type 2 diabetic rats. Phytotherapy Research, 22, 1458-1464. doi:10.1002/ptr.2476

[2] Djurhuus, M.S., Henriksen, J.E., Klitgaard, N.A., Blaabjerg, O., Thye-Ronn, P., Altura, B.M., Altura, B.T. and Beck-Nielsen, H. (1999) Effect of moderate improvement in metabolic control on magnesium and lipid concentrations in patients with type 1 diabetes. Diabetes Care, 22, 546-554. doi:10.2337/diacare.22.4.546

[3] Yajnik, C.S. (2001) The insulin resistance epidemic in India: Fetal origins, later lifestyle, or both? Nutrition Reviews, 59, 1-9. doi:10.1111/j.1753-4887.2001.tb01898.x

[4] Jenkins, A.J., Lyons, T., Zheng, D.Y., Otvos, J.D., Lackland, D.T., Mcgee, D., Garvey, W.T. and Klein, R.L. (2003) Serum lipoproteins in the diabetes control and complications trial/epidemiology of diabetes intervention and complications cohort-Associations with gender and glycemia. Diabetes Care, 26, 810-818. doi:10.2337/diacare.26.3.810

[5] Ceriello, A. (2005) Postprandial hyperglycemia and diabetes complications. Is it time to treat? Diabetes, 54, 1-7. doi:10.2337/diabetes.54.1.1

[6] Abe, A., Kawasoe, C., Kondo, Y. and Sato, K. (2003) Enhancement of norepinephrine-induced transient contraction in aortic smooth muscle of diabetic mice. Acta Medica Okayama, 57, 45-48.

[7] Choi, B.G., Vilahur, G., Viles-Gonzalez, J.F. and Badimon, J.J. (2006) The role of high-density lipoprotein cholesterol in atherothrombosis. Mount Sinai Journal of Medicine, 73, 690-701.

[8] Marles, R.J. and Farnsworth, N. (1995) Antidiabetic plants and their active constituens. Phytomedicine, 2, 137-189. doi:10.1016/S0944-7113(11)80059-0

[9] Pari, L. and Umamaheswari, J. (2000) Antihyperglycaemic activity of Musa Sapientum flowers: Effect on lipid peroxidation in alloxan diabetic rats. Phytotherapy Research, 14, 1-3.

doi:10.1002/(SICI)1099-1573(200003)14:2<136::AID-P 


\section{TR607>3.0.CO;2-K}

[10] Ojewole, J.A. (2005) Hypoglycemic and hypotensive effects of Psidiumguajava Linn. (Myrtaceae) leaf aqueous extract. Methods \& Findings in Experimental \& Clinical Pharmacology, 27, 689-695. doi:10.1358/mf.2005.27.10.948917

[11] Chiwororo, D.H. and Ojewole, J. (2008) Biphasic effect of Psidiumguajava Linn. (Myrtaceae) leaf aqueous extract on rat isolated vascular smooth muscles. Journal of Smooth Muscle Research, 44, 217-229. doi:10.1540/jsmr.44.217

[12] Deguchi, Y. and Miyazaki, K. (2010) Anti-hyperglycemic and anti-hyperlipidemic effects of guava leaf extract. $\mathrm{Nu}$ trition \& Metabolism (Lond), 7, 9. doi:10.1186/1743-7075-7-9

[13] Obatomi, D.K., Bikomo, E.O. and Temple, V.J. (1994) Antidiabetic properties of African mistletoe in streptozotocin-induced diabetic rats. Journal of Ethnopharmacology, 43, 13-70. doi:10.1016/0378-8741(94)90111-2

[14] Cheng, F.C. and Shen, S.C. (2009) Effect of guava (Psidiumguajava L.) leaf extract on glucose uptake in rat hepatocytes. Journal of Food Science, 74, H132-H138. doi:10.1111/j.1750-3841.2009.01149.x

[15] Sunagawa, M., Shimada, S., Zhang, Z., Oonishi, A., Nakamura, M. and Kosugi, T. (2004) Plasma insulin concentration was increased by long-term ingestion of guava juice in spontaneous non-insulin-dependent diabetes mellitus (NIDDM) rats. Journal of Health Science, 50, 674678. doi:10.1248/jhs. 50.674

[16] Olatunji-Bello, I., Odusanya, A.J., Raji, I. and Ladipo, C.O. (2007) Contractile effect of aqueous extract of Psidiumguajava leaves on aortic rings in rat. Fitoterapia, 78, 241-243. doi:10.1016/j.fitote.2006.11.007

[17] Saunders, J.P., Donner, T.W., Sadler, J.H., Levin, G.V. and Makris, N.G. (1999) Effects of acute and repeated oral doses of D-tagatose on plasma uric acid in normal and diabetic humans. Regulatory Toxicology and Pharmacology, 29, S57-S65. doi:10.1006/rtph.1998.1264
[18] McGregor, D.D. (1965) The effect of sympathetic nerve stimulation of vasoconstrictor responses in perfused mesenteric blood vessels of the rat. Journal of Physiology, 177, 21-30.

[19] Suntornsuk, L., Gritsanapun, W., Nilkamhank, S. and Paochom, A. (2002) Quantitation of vitamin C content in herbal juice using direct titration. Journal of Pharmaceutical and Biomedical Analysis, 28, 849-855. doi:10.1016/S0731-7085(01)00661-6

[20] Soltani, N., Keshavarz, M. and Dehpour, A.R. (2007) Effect of oral magnesium sulfate administration on blood pressure and lipid profile in streptozotocin diabetic rat. European Journal of Pharmacology, 560, 201-205. doi:10.1016/j.ejphar.2006.12.020

[21] Soltani, N., Keshavarz, M., Sohanaki, H., ZahedAsl, S. and Dehpour, A.R. (2005) Relaxatory effect of magnesium on mesenteric vascular beds differs from normal and streptozotocin induced diabetic rats. European Journal of Pharmacology, 508, 177-181.

[22] Soltani, N., Keshavarz, M., Sohanaki, H., Dehpour, A.R. and ZAhediAsl, S. (2005) Oral magnesium administration prevents vascular complications in STZ-diabetic rats. Life Science, 76, 1455-1464.

[23] Altura, B.M. and Altura, B.T. (1995) Magnesium and cardiovascular biology an important link between cardiovascular risk factors and atherogenesis. Cellular and Molecular Biology Research, 41, 347-359.

[24] Altura, B.M. and Altura, B.T. (1991) Cardiovascular risk factors and magnesium: Relationships to atherosclerosis, ischemic heart disease and hypertension. Magnesium and Trace Elements, 10, 182-192.

[25] Ozcelikay, A.T., Tay, A., Guner, S., Tasyaran, V., Yildizoglu-Ar, N., Dincer, U.D. and Altan, V.M. (2000) Reversal effects of L-arginine treatment on blood pressure and vascular responsiveness of streptozotocin-diabetic rats. Pharmacological Research, 41, 201-209. doi:10.1006/phrs.1999.0576 